\title{
Determining Anti-Cancer Properties of Novel Tris-Indolyl Amines
}

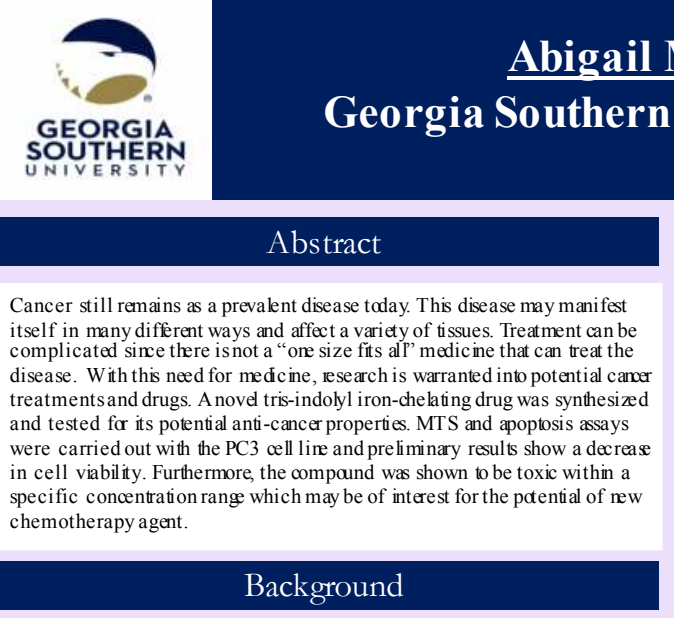

The novel tripodal indlyl amine synthesized, referred to as "Compound B," belongs to a class of molecules called iron chelators. Iron chelators already
have practical application in medicine as a means to remove excess iron in

patients.

Here we investigate potential use of Compound B in cancer treatment. An important aspect of Compound Bis that it is only toxic to cells in a very natiow
range of concentrations. If the concentration increases or decreases, Compour $B$ actually increases cell viability. This shows poential for cancer treatment since the concentration of Compound B would decrease as it diffuses through property of Compound B warrants contined investigation.

\section{pynthesis of Compound $B$}

Synthesis of Compound B

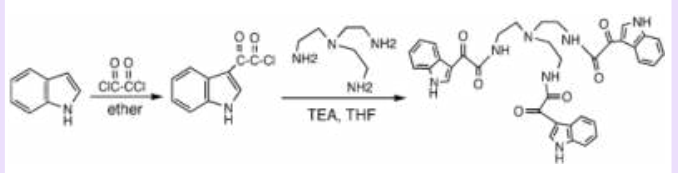

Indole was weighed $(25 \mathrm{~g})$ and placed in a round-bottom flask. To the indole, $(40 \mathrm{~mL}$ ) of diethyl ether was added and stirred in an ice bath until the indole
was dissolved. Oxalyl chloride ( $278 \mathrm{~g}$ ) was added dropwise over 15 min and the reaction mixture allowed to stir for $30 \mathrm{~min}$. An amine mixture consisting of triethylamine (TEA) $(2.2 \mathrm{~g})$, tris(2-aminoethyl)amine (TREN) $(0.87 \mathrm{~g})$, and
THF was made and allowed to chill in an ice bath for $30 \mathrm{~min}$. After the $30 \mathrm{~min}$ the reaction mixture was filtrated and the solid cdlected was placed into another round-bottom flask. The chilled amine solution was added dropwise to filtered. The aqueous solution collected was then recystallized with methano and filtered again to yield a beige solid.

\section{Methods}

\section{Apoptosis Assay}

Cells were plated into a 96 well plate at a concentration of 7,500 cellsper well
and incubated for 24 hoursat $37^{\circ}$ C. Mitoclondrial membrane were labeled via and incubated for 24 hours at $37^{\circ}$ C. Mitochondrial membrane were labeled via
incubation with Mito-tracker red for 30 minutes at $37 \mathrm{C}$. The cells were then
washed and resuspended in celll culture media. Control cells were incubated in washed and resuspended in cell culture media. Control cells were incubated in
the normal cell culture meda. Other cells were treated with either $1 \%$ DMSQ $\mathrm{mM}$ staurosporine, or $74.8 \mu \mathrm{M}$ Compound B. Three samples were taken from each experimental condition at varying time points. Acridine Orange wasused to fluorescently label cellspreviously stained with Mito-tracker red.Cells were also fluorescentl labeled with either Caspase 3, 7 green detection reagert an analysis. Images were captured using a confocal imaging microscope.
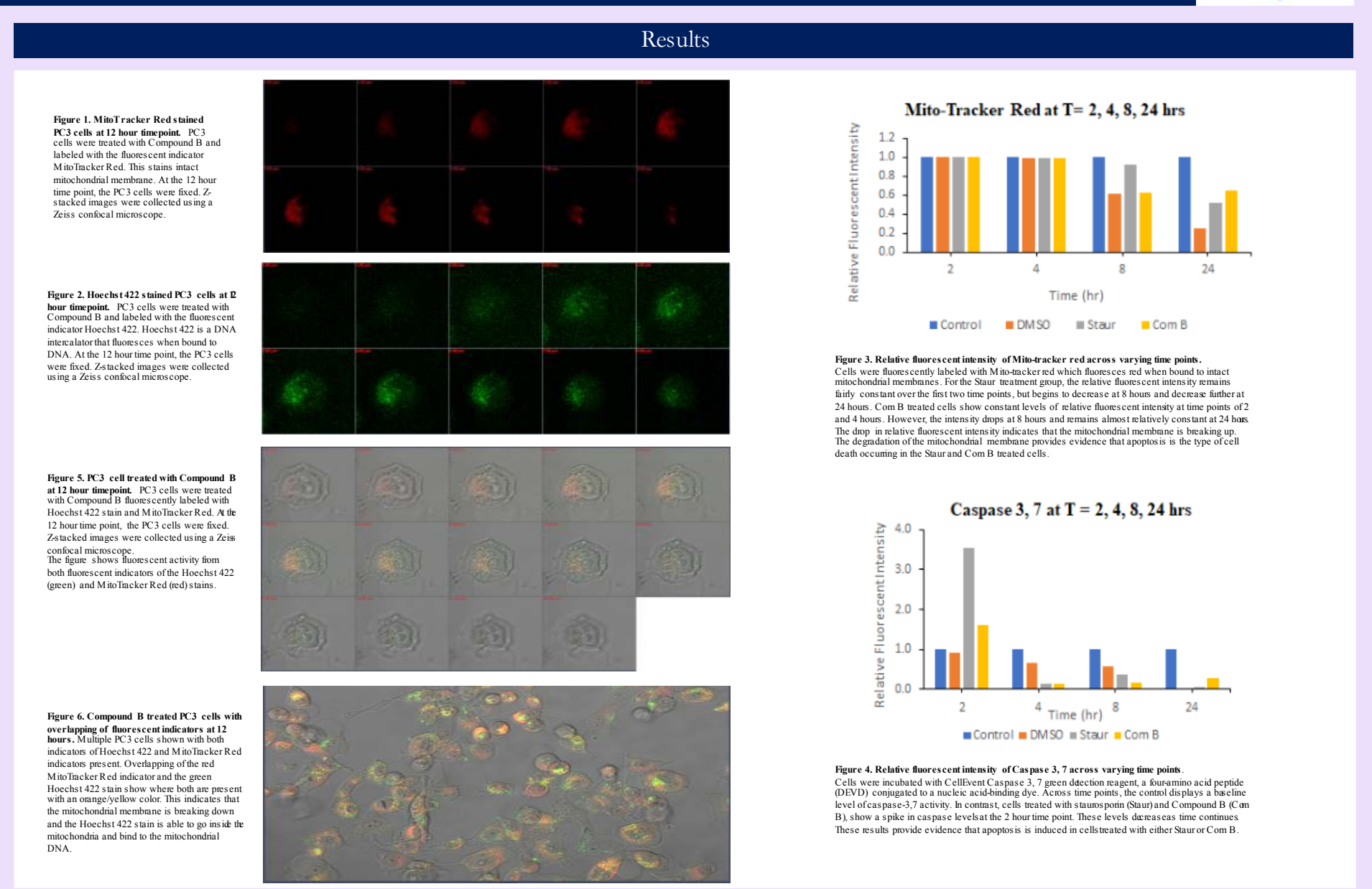

\section{Conclusion and Discussion}

These data provide evidence that Compand B induces apoptosis in $\mathrm{PC} 3$ cells within two hours of treatment, leading to increased levels of cell death between 8 and 24 hours. Figures 3 and 4 show that the type of cell death is apoptosis. The results shown indicate that there is Caspase-3, 7
activity and that the mitochondrial memtrane is breaking down, both of which are elentsin applosis. Hignes 5 and 6 a bo suggest that in both figure isif Hoeckst 422 and MitoTracker $\mathrm{Red}$ wer oc cupring the same bation This then means that bles are appearing in the mitochondria, signaling break down, and the DNA intercal ator Hoechst 422 is able to acess the mitochondrial DNA.
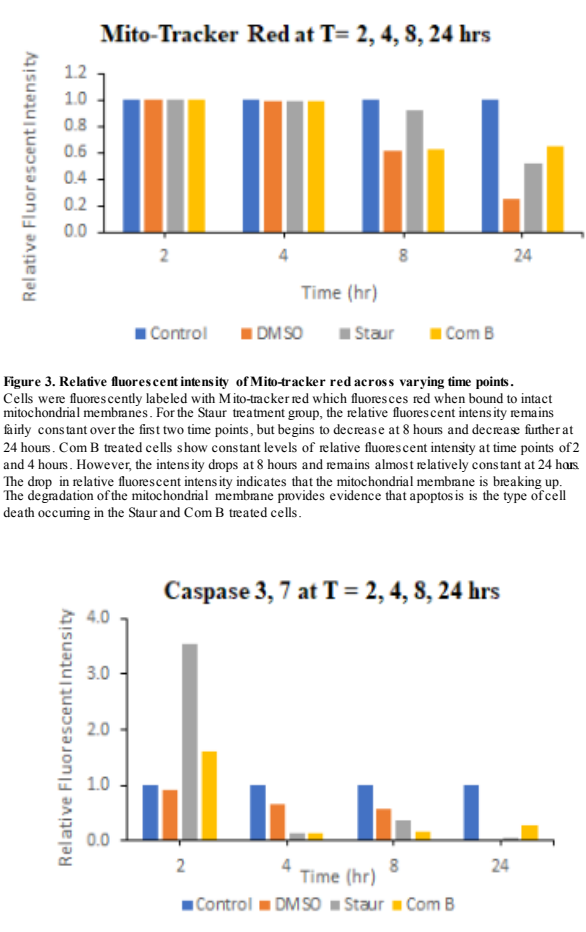

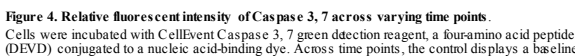

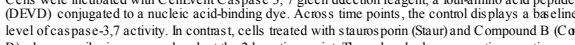

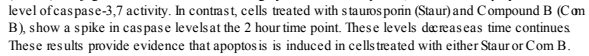

